\title{
Herds of Wildebeest, Flasks of Vodka, Heaps of Trouble: An Embodied Construction Grammar Approach to English Measure Phrases
}

\author{
ELLEN DODGE and ABBY WRIGH'T \\ University of California, Berkeley
}

\section{Introduction}

In this paper we examine English Measure Phrases (EMPs), for example, herd of zebras, cup of coffee, square of cloth, and miles of beach.' Although EMPs internally resemble other phrases of the same form (a noun followed by of followed by a noun phrase), they interact with other elements in the clause in unique ways. On the basis of the phenomena studied in this paper, it will be necessary to distinguish EMPs from other $N$-of-NP phrases. First, EMPs show unusual modificational patterns. Consider the following corpus examples:

(1) The only other colours are provided by a snaking blue-black ribbon of tarmac... (BNC) ${ }^{2}$

(2) Ronni blinked down at the glittering sea that was divided from the craggy landscape by a broad silken ribbon of glittering pale gold sand. (BNC)

In (1) blue-black is understood as modifying tarmac, not ribbon, which serves instead to specify the dimensional boundaries of the tarmac. In (2) broad refers to the dimension of ribbon, while silken describes a quality of the sand.

EMPs also exhibit varying agreement patterns. Consider the following:

(3) To ease the pressure, a truckload of Commandos were taken to the rear, where they could relax for a couple of days. (BNC)

(4) A herd of zebras, hence, produces about a quarter to a third of its weight in prey carcasses per year. (BNC)

\footnotetext{
${ }^{1}$ We would like to thank Charles Fillmore, the FrameNet Project, George Lakoff and Jerry Feldman, the Neural Theory of Language Group, and Andreas Kathol.

${ }^{2}$ For our corpus data we used the British National Corpus (BNC).
} 
In example (4) a herd is construed as singular and shows singular agreement. In sentence (3) the opposite is the case: a truckload of Commandos is construed as plural and shows plural agreement.

Finally, the EMP construction allows for the noun phrase nested inside the EMP to satisfy the selectional restrictions of the verb.

A toddler was fighting for life last night after he swallowed a bottle of lethal acid at a doctor's surgery. (BNC)

In sentence (5) a bottle of lethal acid is an amount of acid; it is unlikely that the child swallowed both the acid and its container. In (6), however, it is clear that the noun phrase, a bottle of champagne, is about a bottle since break's selectional restrictions require that its direct object be solid or a functional item, which can not be satisfied by champagne. In these examples the verb's selectional restrictions can be satisfied by either of the elements in the phrase. Furthermore, EMPs can be nested and the verb can select for the innermost noun. Consider example (7) and note also the plural agreement on the verb; fall agrees with bits, an intervening measure element between load and paper.

(7) When I open it a load of bits of paper fall out and flutter to the ground. (BNC)

We will argue that EMPs can be insightfully analyzed using the fledgling framework of Embodied Construction Grammar (Chang et al. 2001), which we will outline in Section 4. In this form of construction grammar, linguistic constructions map phonological forms to embodied conceptual representations.

\section{English Measure Phrases and Their Function}

As mentioned above, herd of zebras, cup of coffee, square of cloth, and miles of beach are all examples of EMPs. They are of the form $X$ of $Y$, where $X$ is a count noun and $Y$ is a mass noun or plural count noun. We will refer to plural count nouns as multiplex since this captures the undifferentiated nature of the individuals. So for cup of peas, cup will be described as the $X$-element and peas as the multiplex $Y$-element. We will assume a traditional constituent analysis for EMPs. The EMP is a nominal element which consists of a noun modified by a prepositional phrase. We are not making syntactic claims, per se, about the internal form of the phrase (see Akmajian \& Lehrer 1976 and Gawron 2002 for proposals about the internal structure).

What we are calling English Measure Phrases are part of a larger group of constructions sometimes called partitive constructions (Quirk et al. 1985). In this paper, we will not be looking at quality partitive nouns (such as type of font), temporal measures (second of boxing), or phrases that describe part-whole relations (sip of the wine). Actual lexical items in English may appear in more 
than one type of phrase. For example, while hunk of cheese is an EMP, the phrase a hunk of that cheese log describes a part-whole relationship.

We will now propose a typology of EMPs (see Lehrer 1986 and Svensson 1998 for related descriptions). Our typology is constructed on the basis of differences in both the $X$ - and $Y$-elements; types vary as to whether mass and/or multiplex Y-elements are allowed, and also as to what sort of additional classifying information the $X$-element provides. Due to the graded nature of these distinctions, the different types are intended to indicate relevant areas along a continuum rather than being strictly disjoint and distinct types.

\section{$2.1 \quad$ Types of EMPs}

Container-Measures are phrases such as glass of tea, busload of children, or cupful of wine, and can have a Y-element that is either a mass or a multiplex. They constrain and individuate fluids and other entities that must be physically contained to be measured. Information about the typical size of each kind of container gives inexact information about the amount of the Y-element. In this group are also those $X$-elements formed by adding -ful or -load to objects. Objects that are not typically conceptualized as containers usually require this suffix: for example, a handful/*hand of peanuts.

Standard-Measures such as yard of cloth, cup of beans, or gallon of milk are measurements on a standardized scale. These can have either a mass or multiplex entity as their Y-element, though a multiplex can only occur in measures of volume. Standard-Measures provide exact information about the length, area, volume or mass of a substance.

Dimensional-Boundaries are phrases like stick of butter, sheet of paper or ribbon of sand. The $Y$-elements which appear in this type are all internally cohesive and thus are only mass nouns, not plural count nouns. The $X$-element may provide relative size information; for a given substance, $a$ chip is generally smaller than a hunk. Additionally, in the Dimensional-Boundary type of EMP an $X$-element such as stick tells us that the $Y$-element is a fairly rigid, predominantly one-dimensional object (or a long thin thing, as is familiar from classifier languages), thus providing information about shape, dimensionality and rigidity.

The Configuration type has members such as line of trees, circle of crows, stack of papers or heap of stones. In this type, individuals are arranged in a particular configuration, so the $Y$-element must be a multiplex noun. The $X$-element provides information about the configuration's shape and orientation.

The final type is Collection-of-Members which has examples such as herd of zebras, swarm of bees, or team of soccer players and requires a multiplex $Y$-element. In this case the $X$-element provides information about the social or functional relationships between the individuals.

Some lexical items may occur in more than one of the EMP types. For example, a circle of crows is a Configuration since it describes the arrangement of a set of objects in terms of the shape of the boundary, similar to ring of crows; 
however, circle of cloth is of the Dimensional-Boundary type since it indicates that the cloth is a flat object that extends to a circular boundary.

Figure 1: Corpus Examples from the BNC

\section{Container-Measure}

The court also heard from medical experts who found traces of half of a bottle of whisky, and cocaine, in Kin's body. (BNC)

\section{Standard-Measure}

A foot of snow fell in the Scottish Highlands yesterday, bringing chaos to road, rail, and air services. (BNC)

\section{Dimensional-Boundary}

She folded the silver square of wrapping paper in half. (BNC)

\section{Configuration}

A man was sprinkling petrol on a heap of sprouts to help them burn. (BNC)

\section{Collection-of-Members}

You may fatten a couple of pigs to kill and keep a small flock of laying hens or ducks on household scraps. (BNC)

\subsection{The Function of EMPs}

EMPs measure, individuate, and give classificatory information about substances. The $X$-element provides the structure by which an unmeasured and internally undifferentiated $Y$-element is individuated. Although in any given instance a mass noun may be bounded and have a particular size and shape, this information is situational, rather than being an inherent feature of that type of entity. When a substance is simply a mass without any measure or quantification, it is not countable. EMPs serve to make mass nouns countable. Multiplex items are easily quantified through counting, but the count does not supply information about the shape or configuration of the group, the relationships between elements of the group, or even whether or not the multiple entities form a single group at all. In this case the EMP serves to make one countable instance of a multiplex of entities, indicating the relationships between them. Once we have a countable instance of a mass or multiplex, we can describe interactions with that object; this is something that count nouns get for free, but mass nouns do not.

EMPs show significant similarities to the classifier systems of prototypical classifier languages, such as Yucatec Maya (Lucy 1992). Properties provided by the $X$-element in the EMP such as shape, dimensionality, extent, orientation and consistency have been considered universal parameters of classification in studies of classifier systems (Aikhenvald 2000). Additionally, the functions performed by EMPs and classifiers are similar. Lucy $(1992,2000)$ and others have suggested that a classifier form indicates the individuation status (unit or quantity) of a lexical noun indicating type or quality. Under such a view, the nouns in classifier languages are all of the English mass noun type, and require classifiers to be individuated. 
Although similar, there are morphosyntactic differences between prototypical classifiers and EMPs. Prototypical classifiers are closed class items with no independent status, and are used obligatorily. In English there are no individual lexical items dedicated as classifiers; instead, certain lexical items take on a classifying function when present in the EMP (see Lehrer 1986 and Svensson 1998). Furthermore, in English the classifying X-element must appear within a noun phrase with the Y-element, which is not necessarily true in classifier languages. By using a construction grammar approach we can readily recognize the function the $X$-element takes on by becoming a part of this construction.

\section{The Semantics of English Measure Phrases}

As seen in Section 2, our criteria for defining and analyzing EMPs are chiefly semantic. To incorporate these semantic properties into our analysis of EMPs, a more structured account of the meanings involved is needed. In this section we look both at the prototypical scene which motivates EMPs, as well as the underlying cognitive regularities by which we structure such experience.

As noted in the last section, the Y-elements in EMPs and the nouns in classifier languages are structured along parameters which appear to be universals. This is no surprise in an embodied concept of meaning; all human beings share the same perceptual, motor, and cognitive systems and engage in many of the same functional interactions with the world. We suggest that one reason these classification parameters seem largely universal is that classifiers and EMPs both exploit the same basic image schematic structures. We define image schemas as representations of regularities in our perceptual, motor and cognitive systems which structure our experiences and interactions with the world. EMPs exploit a variety of image schemas to provide different sorts of structure. Below is a representation of the Boundary schema, an image schema which provides the structure EMPs utilize to individuate substances.

Figure 2: Schematic Representation of Image Schema

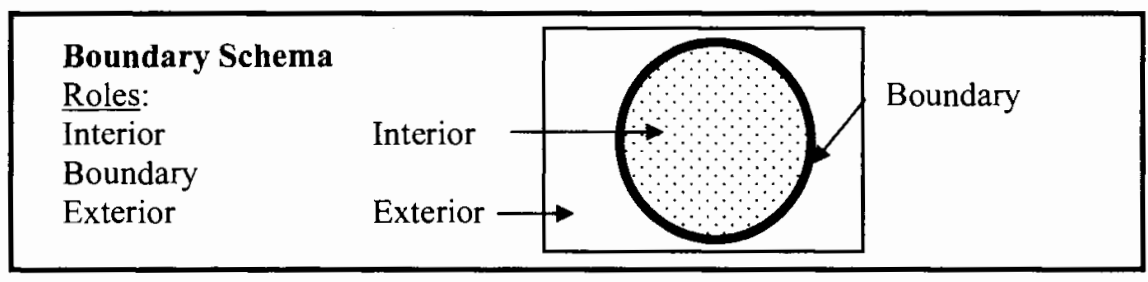

When a substance is individuated, it can be conceptualized as a whole bounded object, with both an interior area and a boundary. The boundary separates the substance from its surroundings, the exterior. Other schemas may combine with the Boundary schema, allowing, for example, the specification of the relative dimensional extent and/or the orientation of the bounded object. 
In addition to image schematic structure, the prototypical scene or frame of measurement which motivates EMPs needs to be described in a structured way. Frame semantics provides such a structure. According to frame semantics, "words represent categorizations of experience, and each of these categories is underlain by a motivating situation occurring against a background of knowledge and experience." (Fillmore 1982: 112). The semantic frame of measurement, or the prototypical measuring scene, includes an agent who partitions and separates a quantity of a substance (mass or multiplex) from an undifferentiated mass in order to handle, transport or otherwise interact with the substance. This is prototypically done using a container, although a physical container need not be present, especially with an internally cohesive mass or arrangement (as in bouquet of flowers or a chunk of cheese). English speakers use in or out with many of these $X$-elements, indicating that they are often construed as metaphorical containers.

(10) He tried to get out of the gang, but was held in by threats of violence.

We cut the bread into chunks.

We put the leaves in a pile.

He divided the old farm into acres to sell to developers.

The idea of containers is a complex one. Most physical containers are artifacts whose primary function is to contain other entities. The container typically acts as a rigid boundary which restrains the contents located in its interior. Fullness plays a role in these containers; flasks can be full of vodka and glasses can be half filled with milk. When one adds more content to the container it becomes fuller but usually not larger. However, some containers have flexible boundaries; they are always completely filled with their contents and grow larger upon the addition of more contents. The boundary of the contained substance is always the same size and shape as the container itself. The metaphorical containers found within this construction are all of this type. One cannot say the full square of cloth, because the square is always completely filled by cloth. Similarly, a gang or herd is completely filled by its members; the addition of more members will increase the size but not the fullness of the group.

Our understanding of EMPs depends on the background knowledge of a measurement frame. Although most previous work on frame semantics has involved verbs which evoke frames that structure entire clauses, frame analyses are also possible for nouns or noun phrases which evoke events and scenes. For most frame-evoking verbs, one or more of the scene's human participants are typically present within the clause. EMPs, however, evoke the measurement frame but do not profile the agents or measurers who are doing the measuring; these measurers are completely absent within the phrase, and may also be absent from the larger clause in which the EMP occurs. 


\section{Embodied Construction Grammar}

We will incorporate both frame semantics and image-schematic structure in our representation of EMPs, to follow in section 5. The framework in which our analysis will be couched is Embodied Construction Grammar (ECG), a grammatical framework being developed by the Neural Theory of Language group at the University of California, Berkeley (Chang et al). ECG builds on earlier work in construction grammar (Goldberg 1995, Kay \& Fillmore 1999, Ginzburg \& Sag 2000) and shares key commitments of other types of construction grammar. As Kay \& Fillmore (1999) describe, "The construction grammarian is required to develop an explicit system of representation, capable of encoding economically and without loss of generalization all the constructions (or patterns) of the language" (p. 2). In construction grammar, then, all patterns of a language are considered constructions and even things as "peripheral" as idioms are worthy of study and have a place within the grammar. Croft (2001) writes: "...construction grammar has generalized the notion of a construction to apply to any grammatical structure, including both its form and meaning" (p. 17).

As described in the introduction, ECG constructions pair form and meaning with the requirement that the meaning component be embodied. This requires that meaning is grounded in the body's perceptual, motor, and non-linguistic conceptual systems, along with world experience. Image schemas and frames are based on our perceptual and motor systems and our experience and are therefore grounded. ECG provides us with a representation that allows us to utilize these concepts and their roles and constraints. Our representation will be chiefly concerned with the semantic properties of EMPs, and we will show that some of their interesting grammatical properties are the result of their semantics. We will analyze EMPs as an instantiation of the EMP Construction, a pairing of form and meaning. The semantic properties of the construction license the internal noun phrase, or $Y$-element, to contribute the semantic category and number of the entire construction.

\section{The English Measure Phrase Construction}

As shown in Section 1, EMPs behave unusually with respect to their interactions with other elements of the grammar. Our ECG account of the EMP Construction provides crucial insight into this behavior. The semantics of the EMP are represented within the construction as a series of constraints that enforce the relationships between the two elements of the constructions. As seen in our representation of the EMP Construction in Fig. 3, below, this construction evokes the measures relation, which has two roles. The Measure is a subcase of Container, as described in Section 3. The Substance must be either a mass or a multiplex. The measures relation, the central relation of the construction, evokes two other relations, fills and contains. The Substance fills the Measure and extends to the boundaries of the Measure as understood through the measurement scene where an agent uses $X$ to measure $Y$. At the same time the Measure contains 
the Substance. These relations taken together allow the Measure to provide shape and amount information for the Substance. In the Constraints section, the $X$-element is bound to the Measure role and the $Y$-element to the Substance role. The Category of the EMP is supplied by Substance; because the phrase describes an amount of a Substance, the phrase is about that Substance. The relations between Measure and Substance are made explicit by showing the bindings of each of their roles to one another. For example, Measure has a Boundary role, referred to as Measure.Boundary, and it is bound to Substance.Boundary.

Figure 3: Semantics of the EMP Construction.

\begin{tabular}{|c|c|}
\hline EMP Const & tion \\
\hline Evokes: & $\begin{array}{l}\text { measures Relation (which in turn evokes contains and fills } \\
\text { relations) }\end{array}$ \\
\hline Roles: & Measure is a subcase of Container \\
\hline & Substance is a mass or multiplex \\
\hline Relations: & Substance fills Measure \\
\hline & Measure contains Substance \\
\hline Constraints: & Substance $\leftarrow$ Y-element \\
\hline & Category of EMP $\leftarrow$ Substance \\
\hline & Measure.Contents $\leftrightarrow$ Substance (because of contains relation) \\
\hline & Measure.Boundary $\leftrightarrow$ Substance.Boundary (from measures) \\
\hline & $\begin{array}{l}\text { Measure.Area|Volume } \leftrightarrow \text { Substance.Amount (from fills) } \\
\text { Measure.DimExt } \leftrightarrow \text { Substance.DimExt }\end{array}$ \\
\hline
\end{tabular}

Thus our construction consists of two entities, the Measure and the Substance, and the relationship between them. Both of the entities play a crucial role in understanding the semantics of the construction. Because the Substance is the element that provides substance properties, it is what the entire construction is "about", which leads to the unusual circumstance where the $Y$-element becomes the category determinant of the entire noun phrase. The $X$-element contributes purely schematic, classificatory information.

\section{Grammatical Repercussions}

Next we will examine what insights the above constructional analysis affords us regarding the unusual grammatical behaviors of the EMP: the unusual selectional restrictions, the modificational patterns and the agreement properties described in Section 1 .

There appear to be two form-identical constructions with different grammatical properties (see examples (5) and (6)). Some of these phrases made our work at the FrameNet Project ${ }^{3}$ difficult. For example, drink might be followed

\footnotetext{
${ }^{3}<$ http://framenet.ICSI.berkeley.edu/ framenet $>$ See Fillmore \& Sato and Baker \& Ruppenhofer, this volume, for more information on the FrameNet Project.
} 
by a bottle of champagne; thus the semantic argument of drink was instantiated within the noun phrase, a bottle of champagne. This construction, as well as the one in example (5), is what we have described as the EMP Construction. The second, seen in (6), is at least superficially a more straightforward prepositional phrase modificational construction, which describes a container and its contents. In order to highlight the grammatical peculiarities of the EMP Construction, it will be instructive to compare these two constructions.

First we will turn to the selectional restrictions. The phrase bottle of champagne can describe either a bottle that is filled with champagne, or a specified amount of champagne. Consider the nearly minimal pair:

The partygoers drank a bottle of champagne.

(14) The partygoers broke a bottle of champagne over the prow of the ship.

Here drink selects for champagne, and break selects for bottle. In (13) champagne is within the noun phrase that serves as the direct object, yet it contributes the semantic category of the entire phrase. In example (14), however, the semantic category of the phrase is contributed by bottle, not champagne, and it thus falls outside our delineation of EMPs. We found no attested examples in the British National Corpus that require bottle of champagne (or any other similar container phrase) to simultaneously describe a bottle and champagne. We do not find, for example, He broke and then wiped up a bottle of champagne. Furthermore such sentences seem to be unacceptable for native speakers. Thus, we can assert that the EMP Construction is a separate construction, which licenses the $Y$-element to contribute the semantic category of the entire phrase. This is a property of this particular construction and is motivated by the semantic properties described in Section 3. As stated in the previous section, the $X$-element serves to provide a boundary for the $Y$-element and the $Y$-element fills the $X$-element, as seen in the prototypical measurement scene; thus the phrase describes an amount of $Y$. This licenses $Y$ to contribute the semantic category of the entire phrase. Thus, the $Y$ element is what predicates will be sensitive to when selecting their arguments.

Secondly, there are interesting alternations between the EMP and other $N$-of$N P$ constructions that involve modificational patterns. Consider the following sentences:

*the Winesap growers of our apples/the growers of our Winesap apples $I$ ate a delicious can of peas/I ate a can of delicious peas.

I smashed a ?*delicious/rusty can of peas.

*I ate a rusty can of peas.

It appears that modifiers of the $Y$ (such as delicious) are licensed to appear at the periphery of the phrase only in the EMP construction (examples (15) and (16)). This property of the EMP is reducible to the same constructional description that explains the selectional restriction facts. The grammar employs a modificational 
construction (since all syntactic patterns, idiomatic or regular, are constructions), which will interact appropriately with the EMP Construction. In examples (16) and (17), delicious is therefore an acceptable modifier in the EMP construction which is about peas, but not in the prepositional phrase modificational construction, which is describing a can. Furthermore, as argued in Section 5, the $X$-element of the EMP provides amount and shape information, but cannot contribute its own physical object properties. Hence, modifiers of physical properties of the container are unacceptable, as shown in example (18).

The final interesting grammatical fact of EMPs is their agreement properties. Recall examples (3) and (4). In (3), truckload of commandoes triggers plural agreement and is the antecedent of a plural pronoun. However in (4), herd triggers singular agreement on the verb and is the antecedent of a singular pronoun. Thus, the agreement is sensitive to something besides the head noun (the $X$-element and what might be called a "syntactic head") of the EMP, since in both cases the $X$ is singular but the EMP is conceived of as plural in one case. ${ }^{4}$

An initial hypothesis might be that the Y-element's contribution of the semantic category of the whole referent will account for the plural number; however, this proves insufficient. It is not the case that singular agreement requires that the singular noun, $X$, be contributing the category of the EMP. In (19), the phrase is about zebras.

\section{The herd of zebras is/are grazing.}

However, when the head noun determines the category of the phrase, it requires singular agreement; this results in the usual case in English grammar where agreement, syntactic head, and category determinant all rely on the same element of the phrase. Plural agreement can be licensed by the EMP construction. In (20) for the predicate are growing to be acceptable requires the EMP construction, in which the phrase is "about" zebras.

(20) The herd of zebras is/*are growing. (where growing refers to herd)

Furthermore, the particular predicate and the nature of the measure seem to make a difference in ease of plural construal for the entity. When the actions are easily understood as applying to each individual, plural agreement is more readily acceptable for most speakers than in cases where the action affects the entire group, as illustrated in (21). When $X$ describes a collection, plural agreement is more readily acceptable as well, as illustrated in examples (22) and (23).

An entire herd of elephants was/were shot.

\footnotetext{
${ }^{4}$ Since our data are from the British National Corpus, some readers might suspect the agreement facts are an artifact of British English's acceptance of plural agreement in sentences that American speakers find clearly unacceptable, as in The government were relieved to balance the budget. However, as examples (3) and (4) show, both patterns of agreement were found in the corpus.
} 
A herd of zebras was/were grazing

A bag of peas was/?were spilled.

Consider Figure 4. The varying conception of the EMP as a singular or plural entity can be understood as a matter of construal sensitive to two properties of the EMP construction. The EMP construction serves to make an individual entity out of a mass or multiplex on the one hand; while on the other hand allowing the phrase as a whole to have the semantic category of the $Y$-element. Thus the individuation properties of the EMP construction and the licensing of the increased importance of $Y$ (to the extreme of the phrase now being about $Y$ ) conflict in determining which entity involved in the construction will determine the number of the entire phrase. This licenses the speaker to construe the EMP with either number. The entity described by the EMP can be construed as collection of undifferentiated individuals or as salient individuals in a group. The latter construal occurs when the $X$ has enough collection properties to make a plural construal possible and, of course, when the $Y$ is a multiplex ${ }^{5}$.

Figure 4: Number Represented as Construal ${ }^{6}$

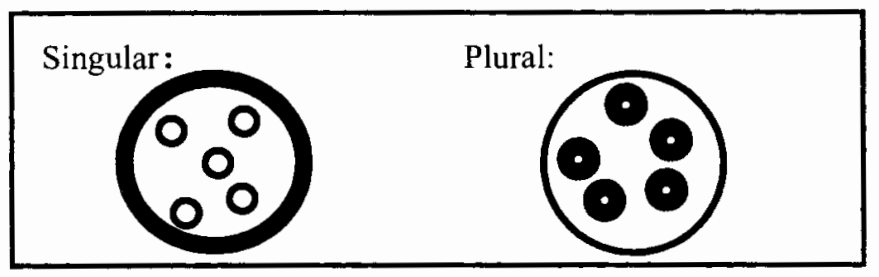

\section{Conclusion}

In conclusion, we have argued that English Measure Phrases instantiate a particular grammatical construction: the EMP Construction. This construction licenses the $Y$-element to contribute the category determinant for the entire phrase, resulting in the case where the Y-element satisfies the predicate's selectional restrictions, and modifiers of the Y-element are licensed to appear at the periphery of the phrase. Furthermore, agreement was argued to be a matter of construal, licensed by the two facets of the EMP Construction: its function of individuating mass substances and its possessing the category of the Y-element, which can consist of a multiplex. We couched our analysis in Embodied Construction

\footnotetext{
${ }^{5}$ It might be suggested that plural agreement is the result of adjacency facts, with the closest noun phrase determining the number agreement on the verb. In (3), however, the appearance of they later in the sentence indicates that the entity a truckload of Commandoes is being conceived as a plural entity. We would not argue, of course, that adjacency effects play no role in agreement; simply that they cannot be responsible for all unusual agreement facts.

${ }^{6}$ Here we follow Langacker 1991, where nouns designate regions and count nouns designate bounded regions.
} 


\section{E. Dodge and A. Wright}

Grammar and argued for the necessity of a framework that recognizes the primacy of constructions and the embodiment of semantics.

\section{References}

Aikhenvald, Alexandra. 2000. Classifiers: A typology of noun categorization devices. Oxford: Oxford University Press.

Akmajian, Adrian and Adrienne Lehrer. 1976. NP-like quantifiers and the problem of determining the Head of an NP. Linguistic Analysis 2(4): 395-413.

Bergen, Benjamin K. and Nancy C. Chang. 2002. Embodied Construction Grammar in simulation-based language understanding. Technical report TR02-004, International Computer Science Institute., www.icsi.berkeley.edu.

British National Corpus. <http://www.hcu.ox.ac.uk/BNC/>

Croft, William. 2001. Radical Construction Grammar. Oxford: Oxford University Press.

Fillmore, Charles. 1982. Frame Semantics. In Linguistics in the Morning Calm. Seoul: Hanshin.

Gawron, Mark. 2002. Two kinds of quantizers in DP. Linguistic Society of America, Annual Meeting, San Francisco, California.

Ginzburg, Jonathan and Ivan Sag. 2000. Interrogative investigations: the form, meaning, and use of English interrogatives. Stanford: CSLI Publications.

Goldberg, Adele. I995. Constructions: A Construction Grammar approach to Argument Structure. Chicago: The University of Chicago Press.

Kay, Paul and Charles Fillmore. 1999. The what's $x$ doing $y$ construction. Language 75(1): 1-33.

Langacker, Ronald. 1991. Concept, Image and Symbol: The cognitive basis of grammar. Berlin: Mouton de Gruyter.

Lehrer, Andrienne. 1986. English classifier constructions. Lingua 68: 109-148.

Lucy, John A. 1992. Grammatical Categories and cognition. Cambridge:

Cambridge University Press.

Lucy, John A. 2000. Systems of nominal classification: a concluding discussion. In Gunter Senft (ed.) Systems of nominal classification. Cambridge: Cambridge University Press.

Quirk, Randolph, Sidney Greenbaum, Geoffrey Leech, and Jan Svartik. 1985. A comprehensive grammar of the English language. New York: Longman Inc

Svensson, Patrick. 1998. Number and countability in English nouns: an embodied model. Uppsala, Sweden: Swedish Science Press.

1203 Dwinelle Hall

University of California, Berkeley

Berkeley, CA 94720-2650

edodge@socrates.berkeley.edu, acwright@socrates.berkeley.edu 